Revista Brasileira de Farmacognosia Brazilian Journal of Pharmacognosy 22(4): Jul./Aug. 2012

\section{New insights on algal products and bioprospection in Brazil. Pharmaceutical, cosmetic and public health applications}

\author{
Eliane Marinho-Soriano, ${ }^{1}$ Ernani Pinto, ${ }^{2}$ Nair S. Yokoya, ${ }^{3}$ Pio \\ Colepicolo, ${ }^{4}$ Valeria Laneuville Teixeira, ${ }^{5}$ Yocie Yoneshigue- \\ Valentin $^{6}$
}

\begin{abstract}
${ }^{1}$ Departamento de Oceanografia e Limnologia, Universidade Federal do Rio Grande do Norte, Brazil,

${ }^{2}$ Departamento de Análises Clínicas e Toxicológicas, Universidade de São Paulo, Brazil, ${ }^{3}$ Núcleo de Pesquisa em Ficologia, Instituto de Botânica, Brazil, ${ }^{4}$ Departamento de Bioquímica, Instituto de Química, Universidade de São Paulo, Brazil, ${ }^{5}$ Instituto de Biologia, Universidade Federal Fluminense, Brazil,

${ }^{6}$ Instituto de Biologia, Departamento de Botânica, Universidade Federal do Rio de Janeiro, Brazil.
\end{abstract}

This special issue provides the readers of the Revista Brasileira de Farmacognosia with access to selected peer-reviewed papers based on lectures presented at the "Third Workshop of RedeAlgas: Biodiversity, Biotechnology and Sustainable Applications", held at Paty dos Alferes, RJ, Brazil, in July, 2011, and attended by academic scientists, professionals from industry and a significant number of students. These papers nicely reflect the diversity of current research in the field of phycology in Brazil presented at the Workshop, with lectures ranging from the potential uses of bioactive secondary metabolites from algae to the mariculture, ecophysiology and micropropagation of macroalgae.

In the last decades, the use of algae and algal products has undergone a substantial increase in the pharmaceutical, cosmetic, veterinary, nutraceutical, biomedicine, bioenergy, food and agricultural industries and in the public health sector. Secondary metabolites extracted from macroalgae are important precursors of vitamins, lipids, proteins and aminoacids, hormones and other molecules that maintain the physiological equilibrium of humans. The diversity and uniqueness of the structures of many algal metabolites reflect both the diversity of the aquatic environments in which they grow and the unique defense mechanisms developed by algae to protect themselves from predation. The food industry in particular employs a wide range of algal derivatives because they contain high contents of fiber, polyunsaturated fatty acids, polysaccharides, minerals, vitamins and antioxidants. The nutritional value of a given species of algae depends on several characteristics such as size, shape, digestibility and toxicity. Macroalgae are at the base of the food chain, serving as the source of nutrients for a wide range of aquatic organisms, such as mollusks, crustaceans, zooplankton and several species of fish. In impacted marine areas, algae are the most important organisms involved in the bioremediation process. Macroalgae can often metabolize persistent organic pollutants such as pesticides, solvents, organometallic compounds and polycyclic aromatic hydrocarbons and can store enormous amounts of heavy metals in their vacuoles. During photosynthesis, algae consume large amounts of $\mathrm{CO}_{2}$ and release large quantities of molecular oxygen into the atmosphere. In addition, algae are the most important organisms in the marine environment for the assimilation of nitrogen, incorporating it into vital molecules such as aminoacids and nucleic acids. Therefore, macroalgae play a vital ecological role in maintaining the equilibrium of marine environments, in preserving diversity and in mitigating global warming.

Finally, the guest editors thank Professor Frank Quina (Instituto de Química, Universidade de São Paulo) for helpful comments and suggestions in all the papers included in this issue and the support from MCTI, CAPES, CNPq, FAPERJ and FAPESP. 